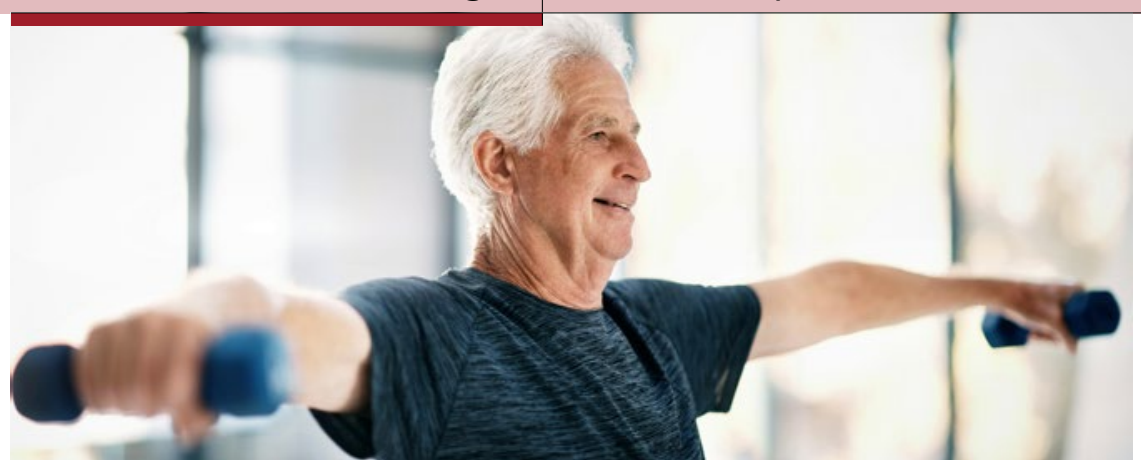

Alter ist nicht unbedingt ein Ausschlusskriterium für eine alloSCT.

res-Überlebenswahrscheinlichkeiten von 48 und $36 \%$. Bemerkenswert ist dabei, dass ca. $85 \%$ dieser Patienten vor der Transplantation nicht in Remission waren.

Was also lernen wir daraus? Heutzutage führen viele Menschen in der 8 . Lebensdekade ein aktives Leben und haben zum Teil Führungspositionen inne. Hämatologische Neoplasien, insbesondere myeloische Leukämien, treten in diesem Alter häufiger auf und können konventionell nur palliativ therapiert werden. Deshalb müsste - eine entsprechende Fitness der Patienten vorausgesetzt - die alloSCT Regelversorgung gerade in dieser Altersgruppe sein.

Für Patienten ab 60 Jahren mit neu diagnostizierter AML nimmt sich die EORTC (European Organisation for Research and Treatment of Cancer) Leukemia Cooperative Group in einer Studie der Frage an, welches die bessere Therapie vor der Transplantation ist: eine konventionelle Induktionsche- motherapie mit Anthracyclin und CytosinArabinosid $(3+7)$ oder die demethylierende Substanz Decitabin („inDACtion vs. induction“; Eudract Nr: 2014-001486-27). Bereits mit der Randomisierung des Patienten nach Diagnosestellung wird eine Spendersuche eingeleitet.

Eine toxizitätsreduzierte Konditionierung für die alloSCT mit 2 Alkylanzien und Fludarabin hat sich bei älteren Patienten bewährt. Es gilt also, bei Diagnosestellung Kontakt mit einem entsprechend erfahrenen Transplantationszentrum aufzunehmen, um den betroffenen Patienten Heilungschancen zu ermöglichen.

\section{Prof. Dr. med. Jürgen Finke}

Klinik für Innere Medizin I,

Klinik für Tumorbiologie,

Universitätsklinikum Freiburg im Breisgau

juergen.finke@uniklinik-freiburg.de

\title{
Kinasehemmer verlängert Leben bei AML
}

Profitieren Patienten mit akuter myeloischer Leukämie (AML) und einer Mutation im FLT3-Gen von einer Zusatztherapie mit Midostaurin? Dieser Frage gingen US-Forscher in einer größeren Studie nach.

E ür ihre Untersuchung haben US-On- kologen 717 AML-Patienten mit FLT3-Veränderungen und einem Alter unter 60 Jahren behandelt. Alle bekamen eine Standardtherapie mit Daunorubicin und Cytarabin sowie 1:1 randomisiert entweder Placebo oder den Multitarget-Tyrosinkinaseinhibitor $\mathrm{Mi}$ dostaurin (50 mg oral 2-mal täglich an den Tagen 8 bis 21).
Die AML-Patienten wurden nach dem Mutationssubtyp in 3 Gruppen eingeteilt: Patienten mit interner Tandemduplikation (ITD) und einem hohen Verhältnis der mutierten zu Wildtyp-Allelen (>0,7; ITD hoch, etwa $30 \%)$, solche mit ITD und einem niedrigen Verhältnis (0,05-0,7; ITD low, etwa $47 \%)$ und solche mit einer Punktmutation in der Tyrosinkinase-Domäne (TDK-Subtyp,

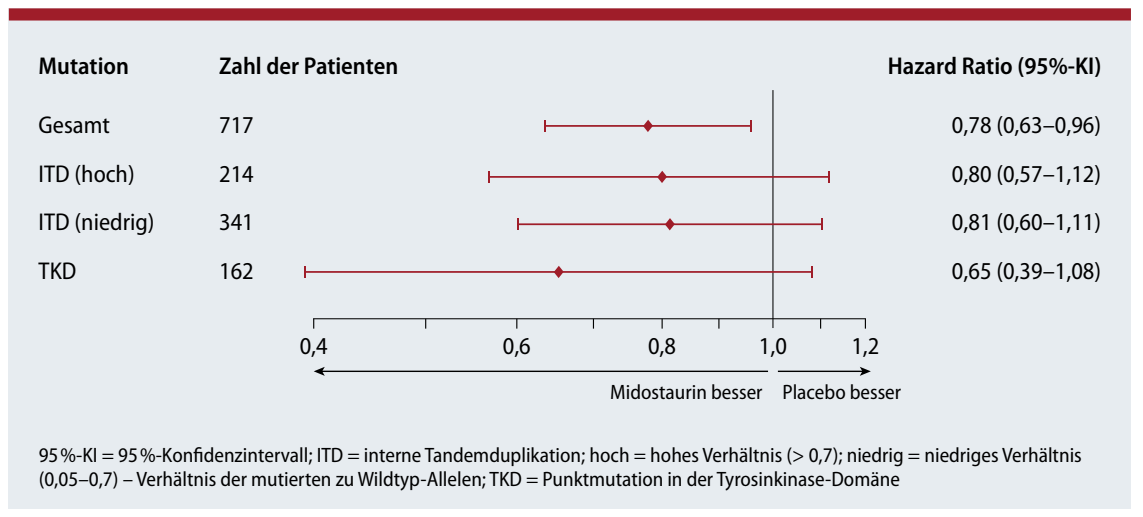

Abb. 1: Subgruppenanalyse zum Gesamtüberleben stratifiziert nach der FLT3-Mutation rund $23 \%$ ). In der Midostaurin-Gruppe war das Gesamtüberleben signifikant länger als in der Placebo-Gruppe (median 74,4 vs. 25,6 Monate; Hazard Ratio für Tod 0,78; $95 \%$-Konfidenzintervall 0,63-0,96; $\mathrm{p}=0,009)$. Nach 4 Jahren lebten unter Midostaurin noch 51,4\%, unter Placebo 44,3\% der Patienten.

Auch hinsichtlich des krankheitsfreien Überlebens profitierten die Patienten von Midostaurin (median 26,7 vs. 15,5 Monate; $\mathrm{p}=0,01)$.

Wenig Differenzen gab es hingegen beim Anteil mit Komplettremission (59\% unter Midostaurin, 54\% unter Placebo) sowie beim Anteil mit allogenen Transplantationen (28\% unter Midostaurin, $23 \%$ unter Placebo). Die Therapieeffekte zeigten sich in allen genetischen Subgruppen. Patienten mit Punktmutationen schienen tendenziell noch etwas stärker vom Multikinasehemmer zu profitieren als die beiden ITD-Subtypen (Abb. 1).

Fazit: AML-Patienten mit einer Mutation im FLT3-Gen profitieren unabhängig vom Mutationstyp von einer Zusatztherapie mit Midostaurin. Thomas Müller

Stone RM et al. Midostaurin plus Chemotherapy for Acute Myeloid Leukemia with a FLT3 Mutation. N Engl J Med. 2017;377(5):454-64. 